\title{
Public Stroke Knowledge: Those Most at Risk, Least Able to Identify Symptoms
}

\author{
Zachary Jarou*, Nathaniel Harris, Liza Gill, Meena Azizi, Shayef Gabasha, Robert LaBril \\ Michigan State University, College of Human Medicine, East Lansing, MI, USA \\ *Corresponding author: Zachary Jarou; zachjarou@gmail.com
}

\begin{abstract}
Background and purpose: Fewer than 1 in 20 patients with acute ischemic stroke are treated with thrombolytic drugs, with threequarters of otherwise eligible patients being excluded secondary to delay in seeking medical treatment. Lack of symptom recognition may contribute to low treatment rates and is an important focus of public health education. The purpose of this study was to determine if an individual's cumulative number of stroke risk factors correlated with their ability to identify stroke symptoms.

Methods: We surveyed adults about their stroke risk factors and knowledge of stroke symptoms at grocery stores and malls in a medium-sized university town in the Midwestern US.

Results: In total, 245 adults completed surveys. Self-reported risk factors included high blood pressure (25\%), high cholesterol (22\%), diabetes (12\%), tobacco use (11\%), alcohol use (7\%), heart disease (7\%), and prior stroke (3\%). Cumulatively, $56 \%$ of respondents had no risk factors, $41 \%$ had 1-3 risk factors, and $4 \%$ had $4+$ risk factors. When administered a six-point stroke symptom knowledge test, respondents with $4+$ risk factors were significantly less knowledgeable, receiving a mean score of 3.2, compared to those with 1-3 risk factors, who scored a mean of 4.6. Those with four or more years of college were significantly more knowledgeable than those with only a high-school education, receiving mean scores of 4.6 and 3.9, respectively. There was no association between stroke knowledge and use of a primary care physician.

Conclusions: Although it is known that individuals with more risk factors are more likely to have a stroke, in our study these respondents were less able to recognize stroke symptoms compared to respondents with fewer risk factors. Future public stroke awareness campaigns should be targeted toward those most at risk so they learn to recognize stroke symptoms and thus seek treatment in a timely manner.
\end{abstract}

Keywords: stroke; CVA; risk factors; warning signs; patient education; public health.

\section{INTRODUCTION}

$\mathbf{S}$ troke is the third leading cause of death and the most common cause of severe, long-term adult disability in the United States. ${ }^{1,2}$ The majority of strokes are ischemic in nature and have the potential to be treated with thrombolytic drugs, improving long-term outcomes when administered to eligible patients in a timely manner. ${ }^{3}$ Despite the availability of this life-improving therapy, fewer than 1 in 20 patients with acute ischemic stroke receive thrombolytics, with three-quarters of otherwise eligible patients being excluded secondary to delay in seeking medical treatment. $^{4-6}$ Of patients delaying treatment, onethird reported that they were waiting to see if their symptoms would improve. ${ }^{5}$ Previous studies have shown that one-quarter to one-third of the public cannot name a single risk factor or warning sign of a stroke. ${ }^{7-9}$ Given these findings, increasing the public's awareness of stroke risk factors, warning signs, and the urgent need for treatment is a public health priority.
The primary purpose of this study is to determine if an individual's cumulative number of stroke risk factors correlates with their ability to identify stroke symptoms. We also examined the relationship between stroke symptom knowledge and age, gender, level of education, and use of a primary care physician. The findings of this study should be considered in developing future stroke awareness campaigns.

\section{METHODS}

\section{Selection and description of participants}

Members of the research team recruited respondents by randomly approaching customers at grocery stores and malls near East Lansing, Michigan. Each respondent was asked if they would be willing to complete a brief survey regarding their knowledge of stroke warning signs. Participants either completed the surveys on their own or gave verbal responses to items read by the research team. Following completion of 
the survey, participants were provided the correct answers to the survey, as well as taught the FAST mnemonic, 'Face, Arm, Speech, Time', endorsed by the American Stroke Association. ${ }^{10}$ Aside from receiving stroke education, respondents received no other compensation for their participation.

\section{Technical information}

This IRB-approved study used an in-person, closedended, 17-item multiple-choice survey. The survey included six knowledge items (stroke symptoms), screening for seven stroke risk factors, and four demographic questions (age, gender, level of education, and use of a primary care physician) (Fig. 1). Knowledge of stroke symptoms was assessed using a portion of the CDC's 2011 Behavioral Risk Factor Surveillance System Questionnaire, ${ }^{11}$ which includes the five signs of stroke used by the National Institute of Neurological Disorders and Stroke. ${ }^{12}$ To guard against patterned responses, we also included chest pain, a non-stroke symptom. Respondents were screened for: high blood pressure, high cholesterol, diabetes, tobacco use, alcohol use, heart disease, and prior stroke.

\section{Statistics}

Prior to analyses, symptoms for which respondents were 'unsure' were combined with 'incorrect' responses to assess for unambiguous symptom knowledge. Risk factors for which the respondent had 'maybe' been diagnosed were coded as being 'present' to include those with borderline risk factors. The cumulative number of risk factors for each respondent was calculated and categorized as 'none', '1-3', or ' 4 or more'. We analyzed the data using Stata v12 (College Station, TX) to estimate the prevalence of each risk factor within the sample population, as well as the frequency of the sample population to unambiguously identify whether each proposed symptom could be considered a warning sign of a stroke. We compared the mean number of correct stroke symptom responses and standard deviations for a number of subgroups including gender, level of education, number of self-reported stroke risk factors, and by use of a primary care physician.

STROKE KNOWLEDGE SURVEY

[Stroke Knowledge]

Please check only one column per symptom.

\begin{tabular}{|r|l|l|l|}
\hline $\begin{array}{c}\text { Which of the following do you think } \\
\text { are symptoms of a stroke? }\end{array}$ & Yes & No & $\begin{array}{c}\text { Don't know } \\
\text { or not sure }\end{array}$ \\
\hline $\begin{array}{r}\text { Sudden confusion or trouble speaking } \\
\text { Sudden numbness or weakness of face, arm, or leg, } \\
\text { especially on one side }\end{array}$ & & & \\
\hline Sudden trouble seeing in one or both eyes & & & \\
\hline Sudden chest pain or discomfort & & & \\
\hline Sudden trouble walking, dizziness, or loss of balance & & & \\
\hline Severe headache with no known cause & & & \\
\hline
\end{tabular}

[Past Medical History]

Please check only one column per risk factor.

\begin{tabular}{|c|l|l|l|}
\hline $\begin{array}{c}\text { Have you ever been told by a health care professional that } \\
\text { you currently have or have had one of the following? }\end{array}$ & Yes & No & $\begin{array}{c}\text { Don't know } \\
\text { or not sure }\end{array}$ \\
\hline High blood pressure & & & \\
\hline High cholesterol & & & \\
\hline Diabetes & & & \\
\hline Prior stroke & & & \\
\hline Heart disease & & & \\
\hline Need to cut down on alcohol use & & & \\
\hline Need to cut down on tobacco use & & & \\
\hline
\end{tabular}

[Demographic Information]

\begin{tabular}{|c|c|}
\hline Age & \\
\hline $\begin{array}{c}\text { Gender } \\
\text { (please circle one) }\end{array}$ & Male or \\
\hline $\begin{array}{l}\text { Highest Level of } \\
\text { Education } \\
\text { Completed } \\
\text { (please circle one) }\end{array}$ & $\begin{array}{c}\text { Never attended school or only attended kindergarten } \\
\text { Grades } 1 \text { through } 8 \text { (Elementary) } \\
\text { Grades } 9 \text { through } 11 \text { (Some high school) } \\
\text { Grade } 12 \text { or GED (High school graduate) } \\
\text { College } 1 \text { year to } 3 \text { years (Some college or technical school) } \\
\text { College } 4 \text { years or more (College graduate) }\end{array}$ \\
\hline $\begin{array}{l}\text { Do you have one or } \\
\text { more person you } \\
\text { think of as your } \\
\text { personal doctor } \\
\text { or health care } \\
\text { provider? } \\
\text { (please circle one) }\end{array}$ & $\begin{array}{c}\text { Yes, only one } \\
\text { More than one } \\
\text { No } \\
\text { Don't know / not sure } \\
\text { Refused }\end{array}$ \\
\hline & Thank you for your participation! \\
\hline
\end{tabular}

Figure 1. Survey instrument. 
Comparison of stroke knowledge by gender and use of primary care physician was performed using a $t$-test. Comparisons of stroke knowledge by level of education and cumulative number of risk factors were performed using ANOVA and Scheffé post-hoc analysis to determine pairwise differences between subgroups. Multivariate regression analysis was also performed to estimate the independent contribution of individual variables on stroke symptom knowledge.

\section{RESULTS}

In total, 245 respondents completed surveys, $58 \%$ of whom were female. The response rate to our survey was not collected; however, very few individuals who were approached refused to participate. The median age of respondents was 40 years, ranging from 18 to 88 years. Forty-nine percent of respondents completed a Bachelor's degree or more, $31 \%$ completed some college or attended technical school, $15 \%$ completed high school or General Educational Development ${ }^{\circledR}$ testing, while $5 \%$ had less than a high-school education.

The self-reported frequency of stroke risk factors in our study population is displayed in Table 1 . More than one in five respondents reported having high blood pressure and/or high cholesterol while roughly 1 in 10 reported diabetes and/or tobacco use. Less than $10 \%$ of respondents reported alcohol use, having a history of heart disease, and/or a prior stroke. Cumulatively, $56 \%$ of respondents had no risk factors, $41 \%$ had $1-3$ risk factors, and $4 \%$ had 4 or more risk factors.

Table 2 summarizes respondents' ability to identify stroke symptoms. The most easily identifiable stroke symptoms were sudden confusion or trouble speaking $(93 \%)$; sudden numbness or weakness, especially on one side (92\%); and sudden trouble walking, dizziness, or loss of balance $(87 \%)$. Fewer respondents were able to recognize sudden headache $(57 \%)$ or sudden trouble seeing in one or both eyes (68\%) as potential stroke symptoms. To guard against patterned re-

Table 1. Individual stroke risk factors present among survey respondents $(n=245)$.

\begin{tabular}{lc}
\hline Risk factors & Present, $n(\%)$ \\
\hline High blood pressure & $61(25)$ \\
High cholesterol & $55(22)$ \\
Diabetes & $30(12)$ \\
Tobacco use & $27(11)$ \\
Alcohol use & $16(7)$ \\
Heart disease & $16(7)$ \\
Prior stroke & $8(3)$ \\
\hline
\end{tabular}

sponses, our survey also included one symptom not related to stroke, sudden chest pain or discomfort, which only $44 \%$ of respondents were able to correctly reject with certainty. Most (85\%) of the respondents reported having a primary care physician.

The subgroup analysis displayed in Table 3 demonstrates that gender and level of education were positively associated, and number of self-reported risk factors negatively associated, with knowledge of stroke warning signs, while use of a primary care physician was not.

Multivariate regression analysis, as seen in Table 4, again demonstrates that level of education was positively associated with stroke knowledge, while cumulative number of self-reported risk factors was negatively associated with stroke knowledge; however, gender was no longer a statistically significant association with stroke knowledge for this study population.

\section{DISCUSSION/CONCLUSIONS}

In this study, we were dismayed to find that respondents with an increased number of stroke risk factors were less able to correctly identify stroke symptoms. This is unfortunate given that this population is most likely to experience a stroke, yet may not understand their need to seek treatment in a timely manner.

We were also surprised to find that there was no association between stroke knowledge and use of a primary care physician; however, other studies have found that only $2 \%$ of patients viewed their physicians as their primary source of stroke knowledge, ${ }^{9}$ so perhaps this is not to be unexpected. This information should however be alarming to primary care providers and efforts should be made to increase stroke education efforts in primary care settings, especially targeting those patients screening positive for four or more risk factors.

It is widely known that socioeconomic position (SEP) is one of the strongest and most consistent predictors of health outcomes. Multiple, often related indicators are commonly used to measure SEP in health research; however, there is no agreement on a single best indicator. While it may sometimes be necessary to capture multiple SEP indicators to avoid residual confounding effects, single indicators are sufficient to demonstrate that a socioeconomic gradient exists. ${ }^{13}$ Income level, race, and level of education have previously been shown to be predictive of stroke knowledge. ${ }^{7,8,14-17}$ For the sake of brevity, we designed our survey to use level of education as a single indicator of SEP. We did not intend to make comparisons between 
Table 2. Ability of survey respondents to identify symptoms of stroke $(n=245)$.

\begin{tabular}{lrr}
\hline Symptom & Correct & Incorrect or unsure \\
\hline Sudden confusion or trouble speaking & $228(93 \%)$ & $17(7 \%)$ \\
Sudden numbness or weakness of face, arm, or leg, especially on one side & $225(92 \%)$ & $20(8 \%)$ \\
Sudden trouble walking, dizziness, or loss of balance & $213(87 \%)$ & $32(13 \%)$ \\
Sudden trouble seeing in one or both eyes & $167(68 \%)$ & $17(32 \%)$ \\
Severe headache with no known cause & $139(57 \%)$ & $106(43 \%)$ \\
Sudden chest pain or discomfort* & $108(44 \%)$ & $137(56 \%)$ \\
\hline
\end{tabular}

*This symptom is suggestive of heart attack, not stroke.

racial or income groups in this study. Similar to the previous work, our results suggest that increased level of education is positively correlated with stroke knowledge. ${ }^{7,8,14,15,18}$

Table 3. Analysis of stroke symptom knowledge by gender, education, number of risk factors, and use of primary care physician.

\begin{tabular}{lcc}
\hline & $\begin{array}{c}\text { Mean correct } \\
\text { responses }( \pm \mathrm{SD})\end{array}$ & $p$ \\
\hline By gender & & \\
Female & $4.6( \pm 1.2)$ & 0.031 \\
$\quad$ Male & $4.2( \pm 1.3)$ & \\
By level of education* & \\
Less than high school & $3.6( \pm 1.5)$ & 0.003 \\
High school or GED & $3.9( \pm 1.5)$ & \\
Some college (1-3 years) & $4.5( \pm 1.1)$ & \\
College graduate (4+ years) & $4.6( \pm 1.2)$ & \\
By self-reported number of risk factors** & \\
$\quad$ None & $4.3( \pm 1.3)$ & 0.002 \\
1-3 & $4.6( \pm 1.1)$ & \\
4 or more & $3.2( \pm 1.6)$ & \\
By self-reported use of primary care physician & \\
Yes & $4.5( \pm 1.2)$ & 0.131 \\
$\quad$ No & $4.1( \pm 1.5)$ & \\
\hline
\end{tabular}

Note: Comparison of stroke knowledge by gender and use of primary care physician was performed using a $t$-test, while comparison by level of education and by number of self-reported risk factors were performed using ANOVA and Scheffé post-hoc analysis.

*Level of education was related to ability to correctly identify stroke warning signs $(F=4.87 ; d f=3241 ; p=0.003)$; Scheffé post-hoc analysis revealed that mean knowledge score for category 'four or more years of college' was significantly different from the 'high school or GED' category ( $p=0.035$ ).

**Increased number of risk factors was also related to ability to correctly identify stroke warning signs $(F=6.26 ; d f=2242 ; p=0.002)$; Scheffé post-hoc analysis revealed that mean knowledge score for category ' 4 or more' risk factors to be significantly different from ' $1-3$ ' risk factors or 'none' categories $(p=0.005$ and $p=0.033$, respectively).

GED, General Education Development ${ }^{\circledR}$ testing.
Female gender has been previously shown to be predictive of increased stroke knowledge. ${ }^{7,8,14,15,18}$ This finding was suggested by our univariate analysis $(p=0.031)$; however, multivariate analysis was not able to confirm this association with the same level of statistical significance $(p=0.057)$. This is likely a type II statistical error, which might have been overcome by increasing our sample size to boost the statistical power of our test.

Discordant with the previous work, our data suggest that there is an overall positive correlation between age and stroke knowledge. This is likely because other research groups have performed analysis using categorical age ranges rather than treating age as a continuous variable. Studies reporting data for multiple age categories have shown that stroke knowledge appears to be at its peak during the middle years of life, ${ }^{17}$ while those using a single age cut-off of 65 to 75 years old have shown the elderly to have decreased stroke knowledge. ${ }^{9,14,18}$

Table 4. Regression analysis to predict stroke symptom knowledge.

\begin{tabular}{|c|c|c|}
\hline Variable & Coefficient* $(\beta)$ & $p$ \\
\hline Age & 0.013 & 0.010 \\
\hline Gender $^{\mathrm{a}}$ & -0.302 & 0.057 \\
\hline \multicolumn{3}{|l|}{ Education $^{\mathrm{b}}$} \\
\hline High school & 0.220 & 0.582 \\
\hline Some college & 0.934 & 0.013 \\
\hline College graduate & 0.958 & 0.008 \\
\hline \multicolumn{3}{|c|}{ Number of risk factors ${ }^{c}$} \\
\hline $1-3$ & 0.206 & 0.254 \\
\hline 4 or more & -0.843 & 0.045 \\
\hline
\end{tabular}

*Estimated regression coefficients are adjusted for all other variables in the table. Adjusted $R^{2}=0.1204$.

'Reference category is 'Females'.

'Reference category is 'Less than high school'.

'Reference category is 'Zero risk factors'. 
Our results suggest that some stroke symptoms are more easily recognizable to the public than others. This is consistent with statewide telephone-based surveys of the Michigan population. ${ }^{8}$ Sudden confusion or trouble speaking and weakness or numbness are the most easily recognizable symptoms of a stroke, while respondents have more difficulty identifying severe headaches, trouble seeing, trouble walking, dizziness, or loss of balance as potentially being warning signs of a stroke. These differences may be related to the symptoms that have been most heavily emphasized in public stroke awareness campaigns such as FAST. ${ }^{10}$

Limitations of this study include the reliance upon respondents to self-report their risk factors, since some patients may have denied risk factors for which they had not actually been screened; responding 'no' rather than 'unsure'. While administering the surveys, a number of respondents stated they were users of tobacco and/or alcohol, however denied this as a risk factor; potentially as an artifact of how the question was framed (Fig. 1). In future studies, it would be helpful to quantify amounts of tobacco and alcohol consumption to eliminate any reporting bias. While cumulative number of self-reported risk factors likely correlated with magnitude of stroke risk, a stroke prediction score such as the Framingham stroke risk profile may have alternatively been used; ${ }^{19}$ however, calculating such a score would require more quantitative data collection than was possible using a brief, public survey format. Given that this survey was administered in grocery stores and malls in a single university town in the Midwest, our results may have limited external validity as a result of selection bias. Indeed many other studies have shown that there are regional differences in public knowledge of stroke warning signs and symptoms. $^{8}$ Additionally, our use of a six-point test to measure symptom knowledge may not necessarily represent clinically meaningful differences in stroke knowledge or predict an individual's response to seek treatment. Individuals with severe symptoms such as the sudden onset of unilateral weakness might seek emergent medical attention regardless of whether or not they knew this was indicative of a stroke. Conversely, individuals with milder stroke symptoms may wait for improvement before seeking treatment.

Compared to previous studies showing that onequarter to one-third of the public cannot name a single stroke symptom, ${ }^{7-9}$ respondents to our survey appeared to have much greater levels of knowledge. This is likely related to the fact that our survey required respondents to accept or reject symptoms from a closed-ended list rather than asking for open-ended responses. Aside from the fact that stroke symptoms may be easier to identify with closed-ended questioning, there may also be an effect of patterned responses. We were surprised to find that more than half of respondents were unable to definitively exclude chest pain as a stroke symptom. Further complicating the use of chest pain to evaluate for the effect of patterned responses is that even with open-ended questioning, the public has difficulty distinguishing between the symptoms heart attack and stroke. ${ }^{15}$ A study of hospitalized patients who had experienced strokes found that only half were able to identify stroke as an injury to the brain. ${ }^{9}$ Despite public confusion in distinguishing between symptoms of stroke and heart attack, it is debatable whether this leads to significant differences in recognizing the need for emergent medical treatment. In future studies using closed-ended questioning, it may be beneficial to increase the number of non-stroke symptoms in order to better evaluate the effect of patterned responses.

Given our findings, future studies should be conducted to better understand why those with the most risk factors may have the least knowledge of stroke symptoms. The lack of association between use of a primary care physician and stroke knowledge should also be further investigated in terms of the quality of primary care received, specifically, but not limited to, the frequency of follow-up visits, continuity with a single provider, and emphasis on patient education. Future public stroke awareness campaigns should be targeted toward those most at risk so that they can seek treatment in a timely manner should they develop any symptoms of stroke onset.

Acknowledgements: This research would not have been possible without the ongoing mentorship of Drs. Henry Barry, MD, Clare Luz, PhD, and Carlos Rios-Bedoya, MPH, ScD, Department of Family Medicine, Michigan State University College of Human Medicine.

Conflict of interest and funding: The authors have no conflicts of interest to report.

\section{REFERENCES}

1. Roger VL, Go AS, Lloyd-Jones DM, et al. 2011. Heart disease and stroke statistics - 2011 update a report from the American Heart Association. Circulation 123:e18-e209. http://dx.doi.org/10.1161/CIR.0b013e3182009701. 
2. Wechsler LR. 2011. Intravenous thrombolytic therapy for acute ischemic stroke. N Engl J Med 364:2138-46.

http://dx.doi.org/10.1056/NEJMct1007370.

3. Hacke W, Kaste M, Bluhmki E, et al. 2008. Thrombolysis with alteplase 3 to 4.5 hours after acute ischemic stroke. N Engl J Med 359:1317-29. http://dx.doi.org/10.1056/NEJMoa0804656.

4. California Acute Stroke Pilot Registry (CASPR) Investigators. Prioritizing interventions to improve rates of thrombolysis for ischemic stroke. Neurology 2005; 64(4):654-9.

http://dx.doi.org/10.1212/01.WNL.0000151850.39648.51. 5. Barber PAM, Zhang J, Demchuk AM, Hill MD, Buchan AM. 2001. Why are stroke patients excluded from TPA therapy? An analysis of patient eligibility. Neurology 56(8):1015-20. http://dx.doi.org/10.1212/WNL.56.8.1015.

6. Katzan IL, Hammer MD, Hixson ED, et al. 2004. Utilization of intravenous tissue plasminogen activator for acute ischemic stroke. Arch Neurol 61(3):346-50. http://dx.doi.org/10.1001/ archneur.61.3.346.

7. Kleindorfer D, Khoury J, Broderick JP, et al. 2009. Temporal trends in public awareness of stroke warning signs, risk factors, and treatment. Stroke 40:2502-6.

http://dx.doi.org/10.1161/STROKEAHA.109.551861.

8. Reeves MJ, Rafferty AP, Aranha AAR, Theisen V. 2008. Changes in knowledge of stroke risk factors and warning signs among Michigan adults. Cerebrovasc Dis 25:385-91. http://dx.doi.org/10.1159/000121338.

9. Kothari R, Sauerbeck L, Jauch E, et al. 1997. Patients' awareness of stroke signs, symptoms, and risk factors. Stroke 28:1871-5. http://dx.doi.org/10.1161/01.STR.28.10.1871. 10. American Stroke Association. Stroke warning signs and symptoms. 2013. Available from: http://strokeassociation.org [cited 10 August 2013].

11. Centers for Disease Control and Prevention. 2011 behavioral risk factor surveillance system questionnaire. 2011. Available from: http://www.cdc.gov/brfss/questionnaires.htm [cited 10 August 2013].
12. National Institute of Neurological Disorders and Stroke (NINDS). Stroke information page. 2013. Available from: http://www.ninds.nih.gov/disorders/stroke/stroke.htm [cited 10 August 2013].

13. Galobardes B, Shaw M, Lawlor DA, Lynch JW, Smith GD. 2006. Indicators of socioeconomic position (part 1). J

Epidemiol Community Health 60:7-12.

http://dx.doi.org/10.1136/jech.2004.023531.

14. Schneider AT, Pancioli AM, Khoury JC, et al. 2003. Trends in community knowledge of the warning signs and risk factors for stroke. JAMA 289(3):343-6.

http://dx.doi.org/10.1001/jama.289.3.343.

15. Reeves MJ, Hogan JG, Rafferty AP. 2002. Knowledge of stroke risk factors and warning signs among Michigan adults. Neurology 59(10):1547-52.

http://dx.doi.org/10.1212/01.WNL.0000031796.52748.A5.

16. Yoon SS, Heller RF, Levi C, Wiggers J, Fitzgerald PE. 2001. Knowledge of stroke risk factors, warning symptoms, and treatment among an Australian urban population. Stroke 32:1926-30. http://dx.doi.org/10.1161/01.STR.32.8.1926.

17. Greenlund KJ, Neff LJ, Zheng ZJ, et al. 2003. Low public recognition of major stroke symptoms. Am J Prev Med 25(4):315-19. http://dx.doi.org/10.1016/

S0749-3797(03)00206-X.

18. Pancioli AM, Broderick J, Kothari R, et al. 1998. Public perception of stroke warning signs and knowledge of potential risk factors. JAMA 279(16):1288-92.

http://dx.doi.org/10.1001/jama.279.16.1288.

19. Wolf P, D'Agostino R, Belanger A, Kannel W. 1991. Probability of stroke: a risk profile from the Framingham study. Stroke 22:312-18.

http://dx.doi.org/10.1161/01.STR.22.3.312. 